\title{
Heartbeat: time to treat the whole patient, not just the valve, when calcific aortic stenosis is present
}

doi:10.1136/heartjnl-2020-318316

Catherine M Otto

A key consideration in timing of aortic valve replacement (AVR) for patients with aortic stenosis (AS) is whether there is an increased risk of sudden cardiac death (SCD) that might be reduced by relief of outflow obstruction. Minners and colleagues ${ }^{1}$ addressed this issue in a retrospective analysis of outcomes in 1840 patients with mild to moderate AS (aortic maximum velocity $2.5-4.0 \mathrm{~m} / \mathrm{s}$ ) in the Simvastatin and Ezetimibe in Aortic Stenosis (SEAS) study. Overall the annualised rate of SCD was $0.39 \%$ per year with 27 events in asymptomatic patients. The most recent echocardiogram prior to SCD showed mild-moderate AS in most (80\%) of these patients with no difference in SCD event rates in those who progressed to severe AS compared to those who did not develop severe valve obstruction. On Cox regression analysis, the only independent risk factors for SCD were age (HR $1.06,95 \% \mathrm{CI} 1.01$ to 1.11 per year, $\mathrm{p}=0.02)$, increased left ventricular mass index (HR 1.20, 95\% CI 1.10 to 1.32 per $\left.10 \mathrm{~g} / \mathrm{m}^{2}, \mathrm{p}<0.001\right)$ and lower body mass index (HR 0.87, 95\% CI 0.79 to 0.97 per $\mathrm{kg} / \mathrm{m}^{2}, \mathrm{p}=0.01$ ) but not the severity of valve obstruction (figure 1 ).

The lack of association between AS severity and the risk of SCD in the SEAS study is thought-provoking and challenges the conventional wisdom that early AVR would prevent SCD in asymptomatic patients with $\mathrm{AS} .^{2}$ In the past, syncope and SCD in patients with AS were thought to be due to mechanisms such as left ventricle (LV) baroreceptor malfunction, hypotension secondary to peripheral vasodilation in the face of fixed valve obstruction, or a shortened diastolic filling interval at high heart rates leading to a reduced stroke volume. However, it is doubtful that any of these mechanisms would account for SCD when AS is only mild to moderate in severity. 'It is increasingly recognised that that AS is not simply a mechanical problem of the valve leaflets not opening fully; instead, AS compromises a complex interplay between the valve,

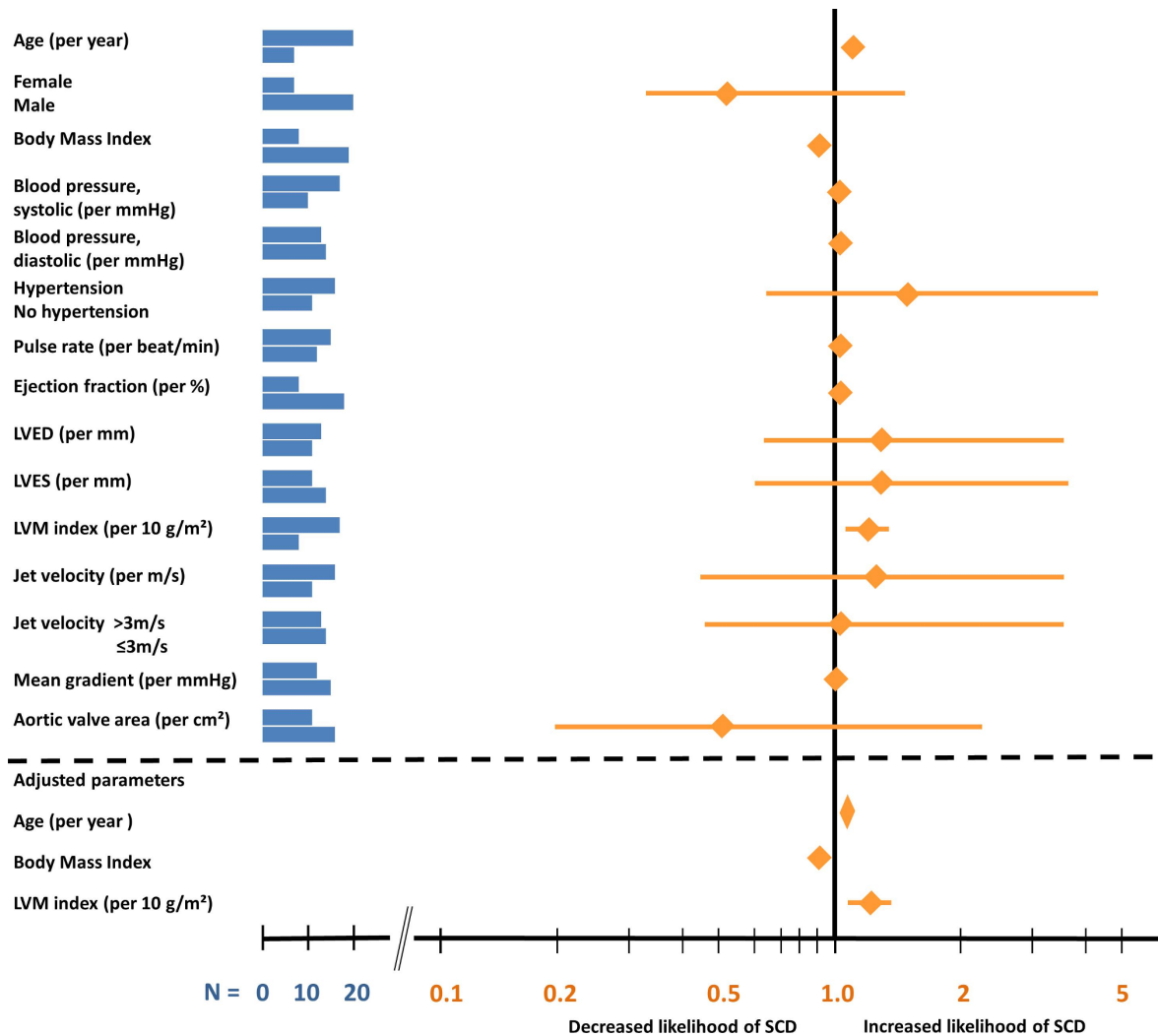

Figure 1 Univariate (top) and multivariate (bottom) Cox regression analyses for SCD during $46.1 \pm 14.6$ months of follow-up in the Simvastatin and Ezetimibe in Aortic Stenosis study. The number of events for each variable is reflected by the dark, horizontal bars with separation at the median for continuous variables. A forest plot visualisation of HRs for SCD is provided on the right. LVED, left ventricular enddiastolic diameter; LVES, left ventricular endsystolic diameter; LVM, left ventricular mass; SCD, sudden cardiac death.
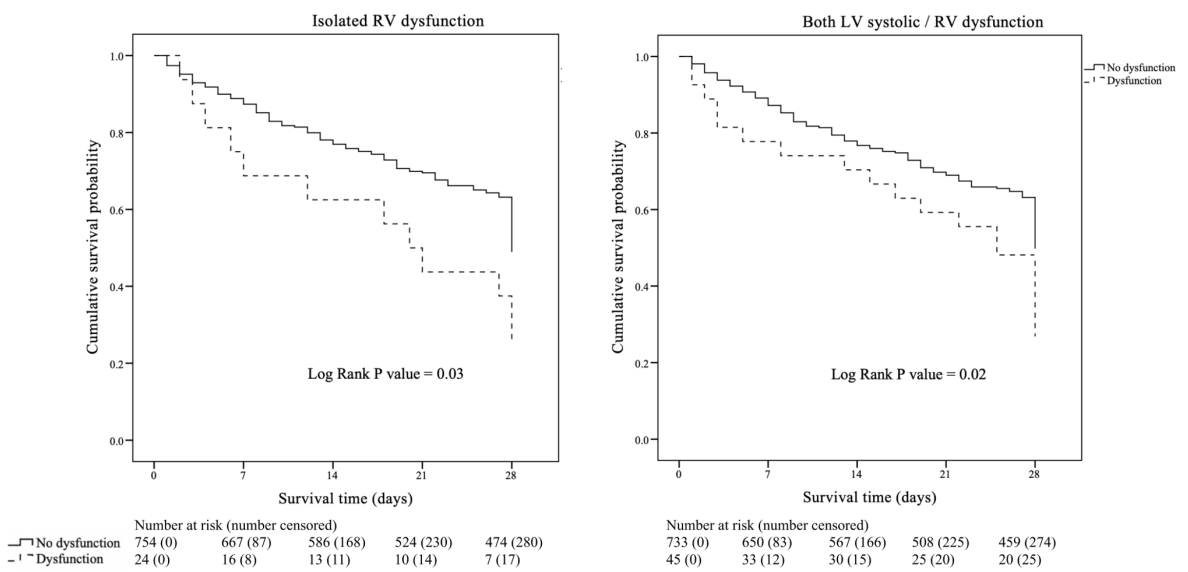

Figure 2 Comparisons of survival curves between each type of dysfunction. LV, left ventricle; RV, right ventricle. 


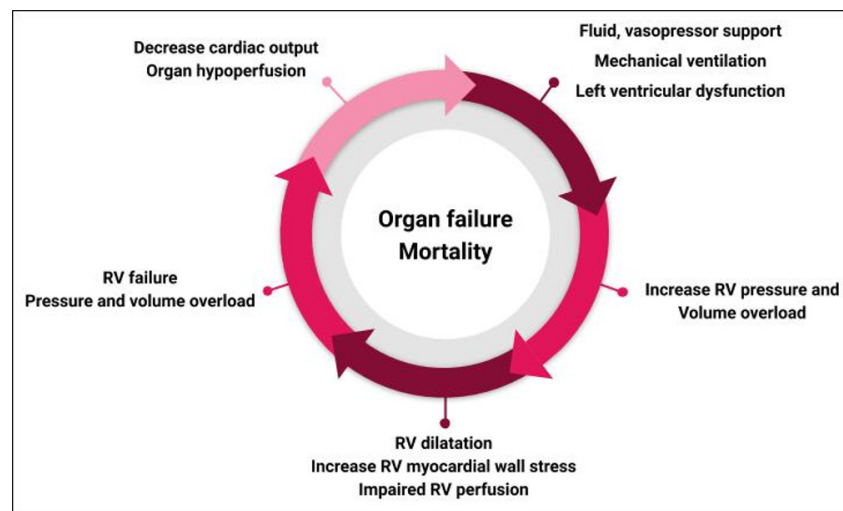

Figure 3 Mechanism of RV dysfunction associated organ failure and mortality in sepsis. RV, right ventricular.

ventricle and vasculature with abnormal function of all three components of the disease process.' As I conclude in an editorial, 'It is unlikely that early AVR will reduce the risk of sudden death when severe valve obstruction is not present. Perhaps it is time to turn our attention to mitigating the non-valvular disease processes in adults with calcific valve disease.'

In another interesting paper in this issue of Heart, Williams and Brown ${ }^{3}$ hypothesised that the apparent benefit of fractional flow reserve (FFR) guidance of percutaneous coronary intervention (PCI) in patients with chronic coronary syndromes (CCS) might simply be due to utilisation of fewer stents rather than to knowledge about the physiological severity of the coronary lesions. In a Monte Carlo simulation using data from the PCI strata of the Bypass Angioplasty Revascularization Investigation 2 Diabetes study, random deferral of PCI progressively reduced the risk of death and myocardial infarction at 1 year, suggesting that FFR-guided deferral of PCI improves outcomes simply because fewer stents are placed.

In an editorial, Weintraub and Boden ${ }^{4}$ put this data into the context of 30 years of clinical trials comparing PCI with optimal medical therapy from CCS and

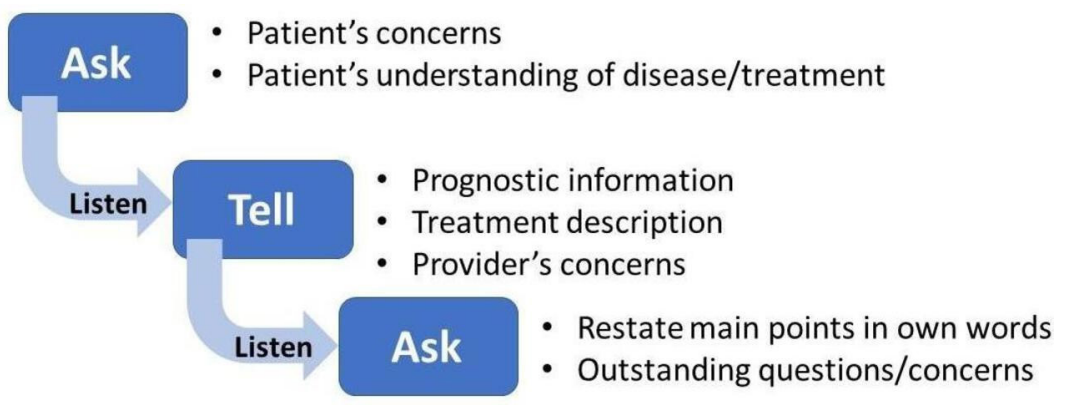

Figure 4 Ask-Tell-Ask tool to guide difficult conversations. of these patients. Any type of sepsisinduced cardiac dysfunction was associated with a significantly higher 28-day mortality (35.9 vs $26.8 \%$; p <0.01), longer intensive care unit length of stay and longer duration of mechanical ventilator, compared with those without cardiac dysfunction. Isolated RV dysfunction was rare (24/270, 8.9\%) but was associated with a higher risk of 28-day mortality (adjusted OR 2.77, $95 \%$ CI 1.20 to $6.40, p=0.02$ ) (figure 2).

The mechanisms of cardiac dysfunction in patients with sepsis are summarised in an editorial by Dugar and Vallabhajosyula ${ }^{6}$ (figure 3). They also point out the challenges in understanding cardiac involvement in patients with sepsis including the effect of timing of imaging on detection, difficulties in measuring RV systolic performance, and differing definitions of RV dysfunction. They conclude: 'there is a crucial need to understand the how to identify RV dysfunction in sepsis and the causative mechanisms associated with higher mortality in this population, which will significantly influence how we prevent and manage this disease process.'

The Education-in-Heart article in this issue by Steiner and Kirkpatrick ${ }^{7}$ focuses on palliative care in management of pateints with cardiovascular disease. Palliative care now encompasses much more than end-oflife comfort measures. Instead, 'Palliative care is a specialised type of medical care that focuses on improving communication about goals of care, maximising quality of life and reducing symptoms' and thus applies to many of our patients at many time points in their disease course. Each of you will want to read the entire article yourself which includes several useful tools, such as the one shown in figure 4, to improve conversations with patients about treatment options, goals of care and planning for adverse outcomes.

Be sure to try the two Image Challenge questions in this issue. ${ }^{89}$ Over 150 boardreview format multiple choice questions based on all types of cardiac images can be found in our online archive on the Heart homepage (https://heart.bmj.com/pages/ collections/image_challenges/).

Funding The authors have not declared a specific grant for this research from any funding agency in the public, commercial or not-for-profit sectors.

Competing interests None declared.

Patient and public involvement Patients and/ or the public were not involved in the design, or conduct, or reporting, or dissemination plans of this research.

Patient consent for publication Not required. 
Provenance and peer review Commissioned; internally peer reviewed.

(C) Author(s) (or their employer(s)) 2020. No commercial re-use. See rights and permissions. Published by BMJ.

\section{Check for updates}

To cite Otto CM. Heart 2020:106:1621-1623.

Heart 2020;106:1621-1623.

doi:10.1136/heartjnl-2020-318316

ORCID ID

Catherine M Otto http://orcid.org/0000-0002-0527-

9392

\section{REFERENCES}

1 Minners J, Rossebo A, Chambers JB, et al. Sudden cardiac death in asymptomatic patients with aortic stenosis. Heart 2020;106:1646-50.

2 Otto CM. Sudden cardiac death in patients with aortic stenosis: maybe it is not the valve? Heart 2020;106:1624-6.

3 Williams C, Brown DL. Effect of random deferral of percutaneous coronary intervention in patients with diabetes and stable ischaemic heart disease. Heart 2020;106:1651--7.

4 Weintraub WS, Boden WE. Deferral of $\mathrm{PCl}$, a safe strategy in diabetic patients with chronic coronary syndromes. Heart 2020;106:1627-8.
5 Kim J-S, Kim Y-J, Kim M, et al. Association between right ventricle dysfunction and poor outcome in patients with septic shock. Heart 2020;106:1665-71.

6 Dugar SP, Vallabhajosyula S. Right ventricle in sepsis: clinical and research priority. Heart 2020;106:1629-30.

7 Steiner JM, Kirkpatrick J. Palliative care in cardiology: knowing our patients' values and responding to their needs. Heart 2020;106:1693-9.

8 Xuereb R, Xuereb S, Xuereb R. A 69-year-old woman presenting with shortness of breath. Heart 2020;106:1637.

9 Marinho AVV, Hidalgo F, Pan M. Rare but unforgettable cause of hypotension. Heart 2020;106:1657. 